\title{
Prävention als Auftrag
}

D ie Prävalenzzahlen atopischer Erkrankungen im Kindesalter und damit auch bei Erwachsenen steigen weiter an. Somit gewinnen qualifizierte Fort- und Weiterbildung zunehmend an Bedeutung. Die (Muster-)Weiterbildungsordnung zum Arzt für Kinder- und Jugendmedizin schreibt zwar den Erwerb allergologischer Kenntnisse und Fertigkeiten vor, allerdings wird heute - wie schon vor 20 Jahren - eine qualifizierte Weiterbildung selten im notwendigen Umfang angeboten.

Vor 20 Jahren wurde in Norddeutschland die „Arbeitsgemeinschaft allergologisch tätiger Kinderärzte e. V.“ gegründet, um im Rahmen von jährlichen Tagungen im Seminarstil den wachsenden Weiterbildungsbedarf in pädiatrischer Allergologie zu decken. Die sehr gute Resonanz führte zur Gründung weiterer Arbeitsgemeinschaften in Süd- und Westdeutschland. In der ehemaligen DDR entstand 1990 die „Arbeitsgemeinschaft Pädiatrische Pneumologie und Allergologie“.

Das Bedürfnis nach Koordination der Aktivitäten der vier Arbeitsgemeinschaften führte 1996 zur Gründung der als Dachverband fungierenden „Gesellschaft für Pädiatrische Allergologie und Umweltmedizin e. V." (GPA), die inzwischen über 1.600 Mitglieder zählt. Seither wird an vielen Orten Deutschlands ein 40 Stunden umfassender "Grundkurs Pädiatrische Allergologie" angeboten, für dessen $16 \mathrm{Mo-}$ dule den Referenten einheitliche Unterrichtsmaterialien zur Verfügung gestellt werden. Weitere Aufgaben sieht die GPA in der Erarbeitung von Positionspapieren und Leitlinien sowie in der Zusammenarbeit mit anderen Fachgesellschaften.

Der Übergang des chronisch kranken Patienten vom Jugendalter ins Erwachsenenalter und der damit verbundene Betreuerwechsel müssen gut vor-

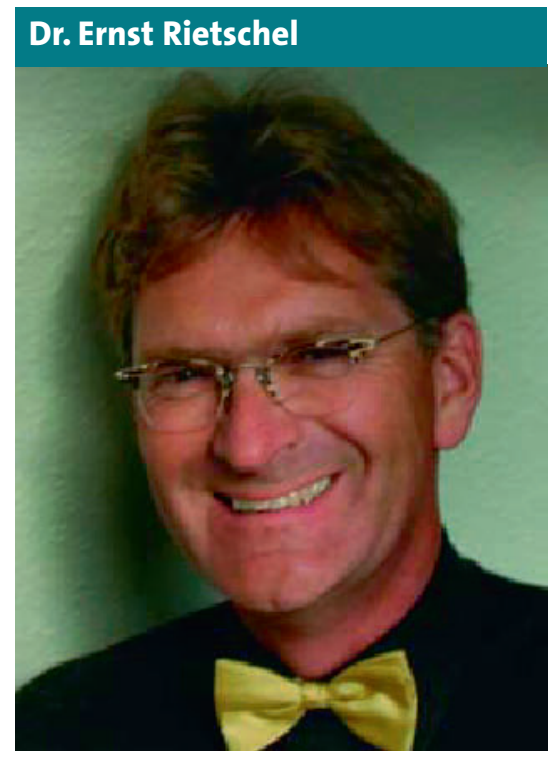

Klinik und Poliklinik für Kinder- und Jugendmedizin, Universität Köln, Sprecher der Sektion Pädiatrie der DGAI, 1. Vorsitzender der GPA

bereitet werden. Pädiater müssen wissen, wem sie ihre Patienten übergeben, „Erwachsenenmediziner" müssen verstehen, woher ihre Patienten kommen. Nur dann kann dieses Vorhaben wirklich zufriedenstellend gelingen.

Hierbei hilfreich sein können gemeinsam erstellte Leitlinien und Positionspapiere der Fachgesellschaften. Das von C. Bauer et al. in dieser Ausgabe veröffentlichte Positionspapier zur „Primären alimentären Prävention" ist der Beginn einer solchen Zusammenarbeit.

B. Niggemanns Artikel zur Sekundärprävention allergischer Erkrankungen mit Hilfe der spezifischen Immuntherapie macht zusätzlich deutlich, dass pädiatrische Allergologen sich besonders für primäre und sekundäre Präventionsmaßnahmen verantwortlich fühlen.

Im Rahmen der tertiären Prävention müssen bei Kindern und Jugendlichen hinsichtlich der Pharmakotherapie eine Reihe von Besonderheiten be- rücksichtigt werden. Bei extern verabreichten Substanzen muss dem im Vergleich zum Erwachsenen größeren Quotienten aus Oberfläche zu Gewicht Rechnung getragen werden, bei der Verordnung systemisch verabreichter Stoffe sind das jeweilige Gewicht und die altersspezifische Pharmakokinetik zu berücksichtigen. Auch sind Sicherheitsdaten aus Studien mit Erwachsenen nicht unmittelbar auf die noch heranreifenden Kinder zu übertragen, insbesondere bei immunologisch wirksamen Therapien.

Kinder bedürfen unserer besonderen Fürsorge, um die altersspezifische psychische und somatische Entwicklung nicht zu gefährden, und um das Risiko für Langzeitnebenwirkungen so gering wie möglich zu halten. M. Kopp hat hierzu die Daten zur Therapie mit Anti-IgE zusammengestellt und deren Einsatz bei Kindern kritisch gewürdigt.

Eine konstruktive Zusammenarbeit der Fachgesellschaften ist aber auch besonders notwendig in gesundheitspolitisch stürmischen Zeiten. ÄDA, DGAI und GPA veranstalten im September in Aachen erstmalig eine gemeinsame Jahrestagung aller deutschen Allergologen (siehe Seite 138), zu der ich Sie jetzt schon ganz herzlich einladen möchte.

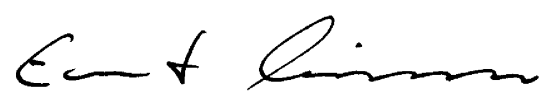

Dr. Ernst Rietschel 